\title{
Morphology of the foramen magnum in syndromic and non-syndromic brachycephaly_authors' reply
}

\author{
Reza Assadsangabi ${ }^{1}$ - Arastoo Vossough ${ }^{1,2}$
}

Received: 7 May 2015 / Accepted: 21 May 2015 / Published online: 26 June 2015

(C) Springer-Verlag Berlin Heidelberg 2015

\section{Dear Editor,}

We would like to thank Drs. Coll and Di Rocco for their comments and have acknowledged their prior longstanding work in the field in our paper. As far as the comments on employing a similar method to their use of non-parametric statistics, actually that was not what was done or intended. After the acquired data did not meet the underlying requirements to use more powerful parametric statistics, such as normality of distribution, etc., unfortunately, we were forced to use non-parametric statistics. And in using non-parametric statistics, we actually used the much more appropriate Kruskal-Wallis statistic with a post hoc test for comparing multiple groups rather than doing multiple paired MannWhitney tests. Assessing orthogonal diameters is quite standard in anatomic measurements of various body regions. Our primary measure was the surface area of the foramen magnum, not the anteroposterior and transverse diameters. Measuring these diameters served mostly to assess the change in shape of the foramen magnum, particularly via changes in the AP-to-transverse ratio. Use of local regression plots is also rather widely used in plotting anatomic growth patterns over time. On the other hand, we completely agree with their conjecture that having a full genetic analysis would result in a more homogenous population and more accurate classification of abnormalities. Unfortunately, not all of our patients had genetic confirmation. Nevertheless, they had been evaluated and diagnosed by an experienced multidisciplinary team at a large referral pediatric craniofacial surgery center. None of the isolated bicoronal synostosis cases were familial.

We are aware of the fact that CSF shunting may lead to secondary closure of some of the calvarial sutures. We did not include these patients in the study as we perhaps thought that this was rather self-evident and therefore regret that it was not explicitly mentioned. As for the statement about age disparity in the study being a limitation, we actually preferred including patients of different ages with their age-matched controls to assess the morphology over a wider age range despite introducing some potential complexity. Also, as for the comment that a smaller foramen magnum being statistically associated with hydrocephalus in Pfeiffer and Crouzon syndromes, but not in Apert syndrome, this is an observation that certainly requires further evaluation and confirmation in future studies. We are happy that our work by and large confirms and extends the work previously done in this area by evaluating a larger group of patients and larger number of disorders, thus adding to the growing literature on complex skull base changes in craniosynostosis.

Arastoo Vossough

vossough@email.chop.edu

1 Department of Radiology, Children's Hospital of Philadelphia, 324 S 34th Street-Wood 2115, Philadelphia, PA 19104, USA

2 Department of Radiology, University of Pennsylvania, Philadelphia, PA, USA 